\title{
Location and Curvature Estimation of Spherical Targets Using Multiple Sonar Time-of-Flight Measurements
}

\author{
Billur Barshan
}

\begin{abstract}
A novel, flexible, three-dimensional multisensor sonar system is described to localize the center of a generalized spherical target and estimate its radius of curvature. Point, line, and planar targets are included as limiting cases which are important for the characterization of a mobile robot's environment. Sensitivity analysis of the curvature estimate with respect to measurement errors and some of the system parameters is provided. The analysis is verified experimentally for specularly reflecting cylindrical and planar targets. Typical accuracies in range and azimuth are $0.17 \mathrm{~mm}$ and $0.1^{\circ}$, respectively. Accuracy of the curvature estimate depends on the target type and system parameters such as transducer separation and operating range.
\end{abstract}

Index Terms - Cylinders, data acquisition, distance measurement, intelligent sensors, radius of curvature estimation, robot sensing systems, sonar measurements, sonar position measurement, spheres, time-of-flight measurement.

\section{INTRODUCTION}

$\mathbf{I}^{\mathbf{N}}$ $\mathrm{N}$ this paper, we present a sonar system capable of estimating the radius of curvature and position of generalized spherical targets. Such systems have applications for intelligent systems, in particular for mobile robots. Although most of today's mobile robots operate in two-dimensional (2-D) environments, three-dimensional (3-D) target recognition and discrimination has potential significance for robots operating in 3-D environments such as airborne or underwater robots. Several researchers have investigated the limitations of sonar for 3-D target recognition and tracking. In [8], an analytical approach to surface curvature extraction is described which employs differential geometry. An acoustic imaging system which combines holography with neural networks for the recognition of 3-D objects is described in [21]. Peremans et al. [17] and Sabatini [19] have both investigated curved reflectors using linear sensor configurations. In [11], the minimum amount of information and actuation needed to track a ball in 3-D has been determined and implemented using qualitative methods only. Kleeman and Akbarally have classified and discriminated the target primitives commonly occurring in 3-D space [10]. Hong and Kleeman treat the classification and localization of 3-D room features using maximum likelihood

Manuscript received June 10, 1996; revised September 16, 1999. This work was supported by TÜBİTAK under Project EEEAG-92 and the British Council Academic Link Program.

The author is with the Department of Electrical Engineering, Bilkent University, 06533 Ankara, Turkey.

Publisher Item Identifier S 0018-9456(99)09592-3.

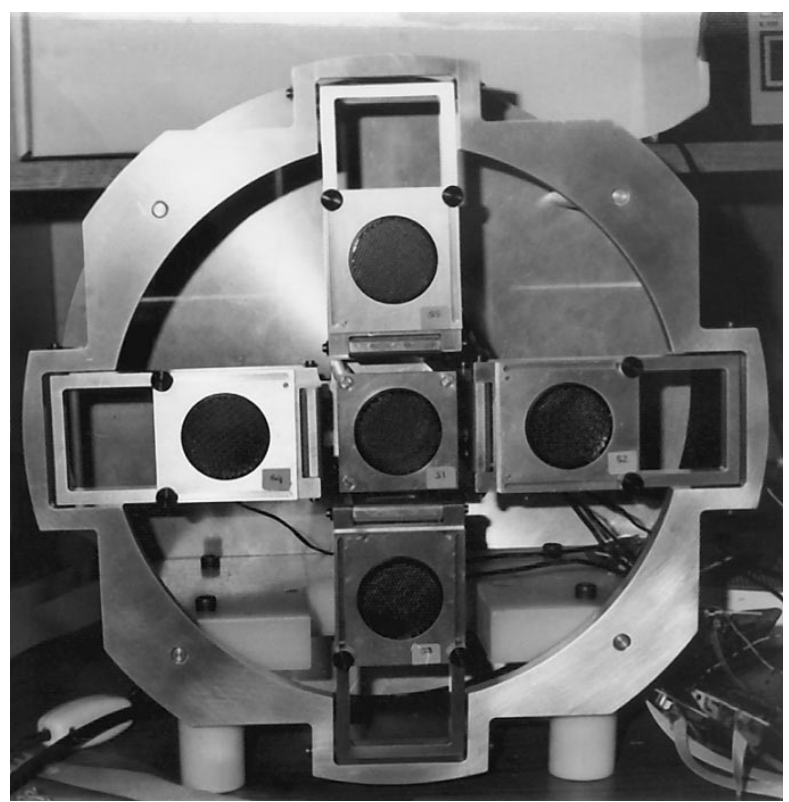

Fig. 1. Sensing system.

estimation and a low-sample rate equilateral triangular sonar configuration [9]. Kuc reports differentiation of O-rings and coins in 3-D using an adaptive sonar configuration [13], [14].

The aim of this paper is to estimate the unknown parameters of spherical targets in 3-D. In the next section, the sensing system is described. In Section III, the geometry of reflection from spherical targets is considered and analyzed. The important limiting cases of point and planar targets are highlighted. Sensitivity analysis of curvature estimation with respect to measurement errors and some system parameters is provided in Section IV. Experimental results which verify the analysis are presented in Section V.

\section{SENSING Device}

The sensing device used in this investigation was precision constructed for 3-D sonar applications. The unit, illustrated in Fig. 1, consists of five Polaroid 6500 series acoustic transducers, each operating at a resonance frequency of $f_{0}=49.4$ $\mathrm{kHz}$ [18]. A central transducer is flanked by four transducers symmetrically. The position of the central transducer is fixed but the separation $d$ of each surrounding transducer from the center can be manually adjusted between 7.5 to $12.0 \mathrm{~cm}$. 


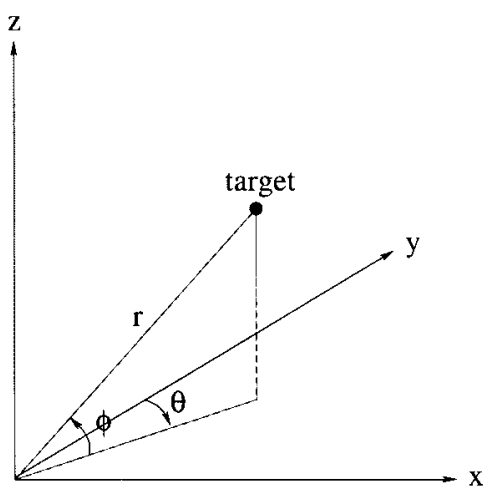

Fig. 2. Geometry of the coordinate system used, illustrating the target range $r$, azimuth $\theta$, and elevation $\phi$. The five transducers are on the $x$ and $z$ axes, one being at the origin and the others symmetrically located around it.

When operated in the pulse-echo mode [18], these devices offer complete flexibility in the firing sequence and timing at which the individual transducers can be fired. In the firing pattern used here, each transducer registers only the echo of the signal transmitted by itself. Assuming the target is stationary, the firing is done sequentially to avoid crosstalk between the transducers. This is geometrically simpler to analyze as compared to firing patterns where transducers simultaneously detect signals transmitted by each other. After each transmission, the detected waveform is recorded and a round-trip time-of-flight (TOF) reading is obtained by thresholding the echo signal as described in [2].

\section{TARget Reflection GeOMEtRy}

In the following analysis, a spherical target of radius $R$ is assumed to be stationary at spherical coordinates $(r, \theta, \phi)$. The coordinate system used is illustrated in Fig. 2. Although this is a nonstandard spherical coordinate system, it is more convenient for explicitly representing the azimuth and elevation angles of the target, as conventionally done in sonar/radar applications.

\section{A. Spherical Target With Radius $R$}

According to the firing pattern described in Section II, the geometry of Figs. 2 and 3 indicates that the distance measurements at the transducers are

$$
\begin{aligned}
& h_{\circ}=\frac{c t_{\circ}}{2}=r-R \\
& h_{r}=\frac{c t_{r}}{2}=\sqrt{r^{2}+d_{r}^{2}-2 d_{r} r \cos \phi \sin \theta}-R \\
& h_{l}=\frac{c t_{l}}{2}=\sqrt{r^{2}+d_{l}^{2}+2 d_{l} r \cos \phi \sin \theta}-R \\
& h_{u}=\frac{c t_{u}}{2}=\sqrt{r^{2}+d_{u}^{2}-2 d_{u} r \sin \phi}-R \\
& h_{d}=\frac{c t_{d}}{2}=\sqrt{r^{2}+d_{d}^{2}+2 d_{d} r \sin \phi}-R
\end{aligned}
$$

where $t_{0}, t_{r}, t_{l}, t_{u}, t_{d}$ are the TOF values obtained at the middle, right, left, up and down transducers respectively, and $c$ is the speed of sound in air. ${ }^{1}$ We will generally concentrate

${ }^{1} c=331.4 \sqrt{\frac{T}{273}} \mathrm{~m} / \mathrm{s}$, where $T$ is the absolute temperature in Kelvin. At room temperature, $c=343.3 \mathrm{~m} / \mathrm{s}$.

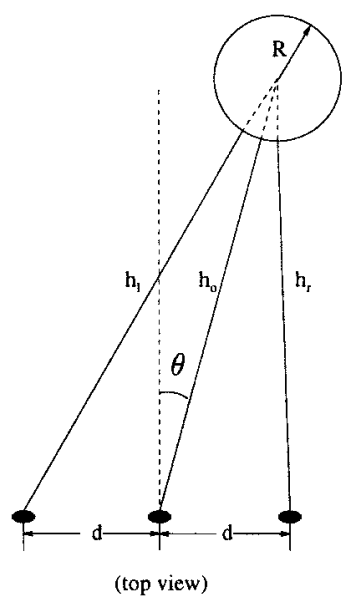

Fig. 3. Geometry of the target position for $d_{r}=d_{l}=d$.

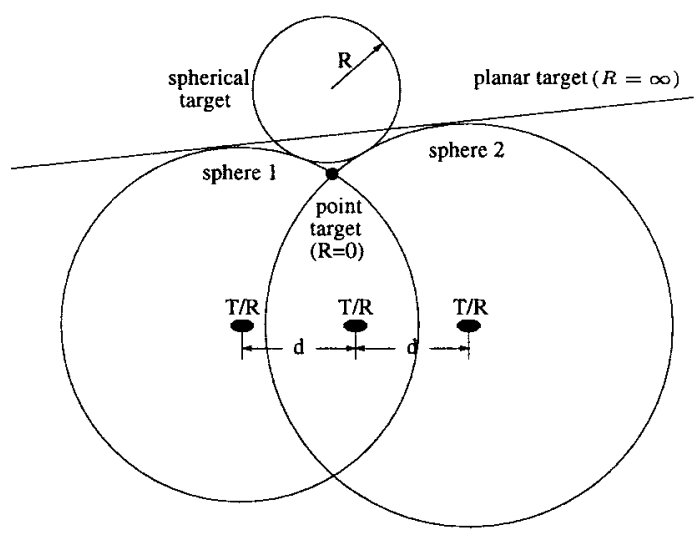

Fig. 4. Indeterminacy of curvature with only two measurements. The unknown target can have any curvature from zero to infinity. Here, $d_{r}=d_{l}=d$.

on the case $d_{r}=d_{l}=d_{u}=d_{d}=d$. However, distinguishing the four transducer separations will be important in the sensitivity analysis. Each measurement confines the possible target locations to a sphere centered at the corresponding transducer. At least three measurements are necessary to identify the curvature of the target both in 2-D and 3-D. This is illustrated in Figs. 4 and 5. Assuming $d_{r}=d_{l}=d_{u}=d_{d}=d$ is exactly known, from the previous equations, $R$ can be solved and expressed in different forms

$$
\begin{aligned}
R & =\frac{\left(h_{r}^{2}+h_{l}^{2}\right)-2\left(h_{\circ}^{2}+d^{2}\right)}{4 h_{\circ}-2\left(h_{r}+h_{l}\right)} \\
& =\frac{\left(h_{u}^{2}+h_{d}^{2}\right)-2\left(h_{\circ}^{2}+d^{2}\right)}{4 h_{\circ}-2\left(h_{u}+h_{d}\right)} \\
& =\frac{\left(h_{u}^{2}+h_{d}^{2}\right)-\left(h_{r}^{2}+h_{l}^{2}\right)}{2\left[\left(h_{r}+h_{l}\right)-\left(h_{u}+h_{d}\right)\right] .}
\end{aligned}
$$

Note that (2) and (3) both require three measurements and the knowledge of $d$, whereas (4) involves four measurements, and not the value of $d$.

Although the above geometry involves five measurements to determine the four unknowns $(r, \theta, \phi, R)$ of the 3-D curvature estimation and localization problem, it should be clear that, in principle, four measurements should be sufficient to determine 


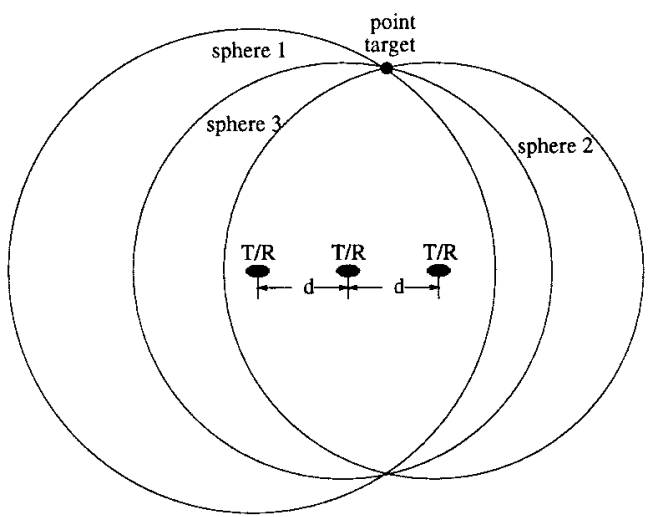

(a)

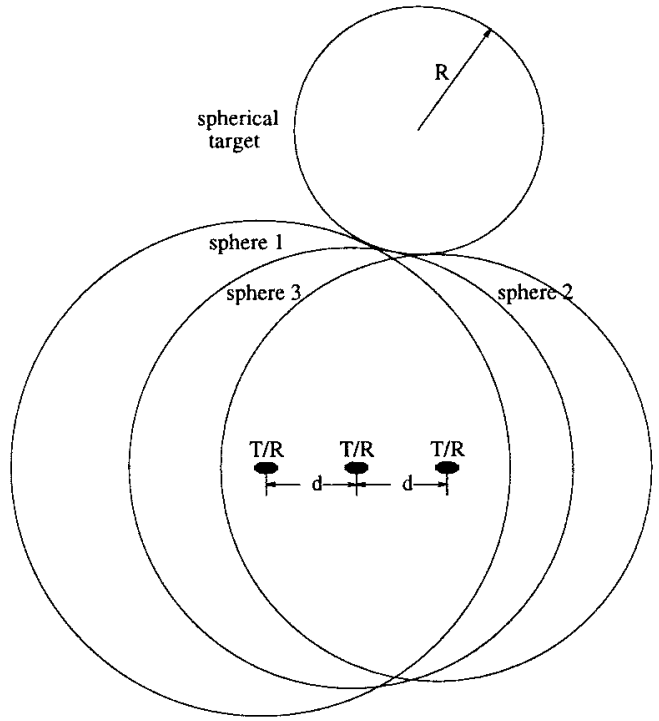

(b)

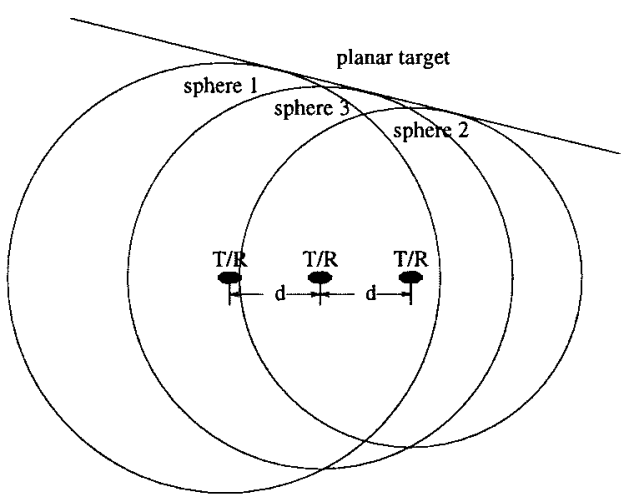

(c)

Fig. 5. Measurement geometry for (a) point, (b) spherical, and (c) planar targets for $d_{r}=d_{l}=d$. Three measurements uniquely identify the curvature of the unknown target both in 2-D and 3-D.

these four unknowns. (In 2-D, three measurements are sufficient to determine the three unknowns.) Since by equating any two of (2)-(4), one of the TOF measurements can be expressed in terms of the other four, one of the five TOF measurements is redundant. It is possible to find an algebraic solution in terms of only four measurements when the transducers are co-planar and symmetrically located at the corners of a square (see the
Appendix) [20]; however, this solution is quite complicated, computationally expensive to evaluate, and prone to rounding error. Addition of the (nominally redundant) fifth measurement leads to the following simpler solution which also exhibits a certain amount of robustness

$$
\begin{gathered}
r=h_{\circ}+R \\
\theta=\sin ^{-1}\left[\frac{\left(h_{l}-h_{r}\right)\left(h_{l}+h_{r}+2 R\right)}{\sqrt{16 d^{2}\left(h_{\circ}+R\right)^{2}-\left(h_{d}-h_{u}\right)^{2}\left(h_{d}+h_{u}+2 R\right)^{2}}}\right] \\
\phi=\sin ^{-1}\left[\frac{\left(h_{d}-h_{u}\right)\left(h_{d}+h_{u}+2 R\right)}{4 d\left(h_{\circ}+R\right)}\right] .
\end{gathered}
$$

\section{B. Point Target: The Limit $R \rightarrow 0$}

In the limit $R \rightarrow 0$, a point target is obtained. Point-target localization in 2-D has been considered in [3] and two methods of estimating the location have been presented using a linear array of transducers. The equations in 3-D derived above for finite $R$ become simpler in the limit $R \rightarrow 0$ :

$$
\begin{aligned}
& r=h_{\circ}=\sqrt{\frac{h_{r}^{2}+h_{l}^{2}}{2}-d^{2}}=\sqrt{\frac{h_{u}^{2}+h_{d}^{2}}{2}-d^{2}} \\
& \theta=\sin ^{-1}\left[\frac{\left(h_{l}^{2}-h_{r}^{2}\right)}{\sqrt{16 d^{2} h_{\circ}^{2}-\left(h_{d}^{2}-h_{u}^{2}\right)^{2}}}\right] \\
& \phi=\sin ^{-1}\left[\frac{\left(h_{d}^{2}-h_{u}^{2}\right)}{4 d h_{\circ}}\right] .
\end{aligned}
$$

In this case, since the measured signal amplitude decreases as the target gets smaller (that is, as $R$ decreases), the region in which the target can be detected is smaller. Characterizing the point-target response of a sensor is important not only for its application to point or edge-like targets, but also to assess its performance on extended targets. There are different approaches to model extended targets [1], [15], [17]. If the approach is one of hypothesis testing or one of parameterizing the extended target, then sensor performance may not be easily related to its point-target response. On the other hand, for extended targets of unknown shape with possible roughness, point-target analysis can be extremely useful [7].

\section{Planar Target: The Limit $R \rightarrow \infty$}

For the limiting case $R \rightarrow \infty$, the target becomes a plane. Both the distance to the center of the target and its radius of curvature become infinity. In this case, either the limits of the above equations can be taken, or more simply, the perpendicular distances of the transducers to the plane can be directly derived from the geometry as

$$
\begin{aligned}
h_{r} & =h_{\circ}-d \cos \phi \sin \theta \\
h_{l} & =h_{\circ}+d \cos \phi \sin \theta \\
h_{u} & =h_{\circ}-d \sin \phi \\
h_{d} & =h_{\circ}+d \sin \phi
\end{aligned} .
$$


The solution is

$$
\begin{aligned}
& r=h_{\circ}+R=\infty \\
& \theta=\sin ^{-1}\left[\frac{h_{l}-h_{r}}{\sqrt{4 d^{2}-\left(h_{d}-h_{u}\right)^{2}}}\right] \\
& \phi=\sin ^{-1}\left[\frac{h_{d}-h_{u}}{2 d}\right] .
\end{aligned}
$$

The distance to the surface of the plane is simply $h_{\circ}$ and the shape of the planar patch that effectively contributes to the signal is approximately a disk of radius $r \tan \theta_{0}$.

\section{SENSITIVITY ANALYSIS}

\section{A. Sensitivity to TOF Measurement Errors}

A sensitivity analysis has been performed to determine how much variation would result in the radius of curvature estimate $R$ as a result of measurement errors and variation in certain system parameters. Since $h_{r}+h_{l}$ and $h_{u}+h_{d}$ are both close to $2 h_{\mathrm{O}}$, the denominators in (2)-(4) are very small, and the value of $R$ computed from these expressions is extremely sensitive to errors in the TOF measurements. This is an intrinsic feature of triangulation with relatively closely-spaced detectors.

By evaluating the partial derivatives of (2) with respect to $h_{\mathrm{o}}, h_{r}$ and $h_{l}$, the total error due to TOF measurement errors can be found as

$$
\Delta R=\frac{\partial R}{\partial h_{\circ}} \Delta h_{\circ}+\frac{\partial R}{\partial h_{r}} \Delta h_{r}+\frac{\partial R}{\partial h_{l}} \Delta h_{l} .
$$

In Fig. 6(a), $\frac{\partial R}{\partial h}$ has been plotted for $r$ between $0-1.5 \mathrm{~m}$. A spherical target with radius $75 \mathrm{~mm}$ is assumed to be stationary at $\theta=\phi=0^{\circ}$. Error sensitivity is greatest for smaller transducer separations as discussed below. Thus, to consider the worst case, we set the transducer separation to $d=7.5$ $\mathrm{cm}$ which is the smallest possible separation in our system. For fixed transducer separation, error in radius of curvature increases nonlinearly with increasing $r$. The typical rangemeasurement-error standard deviation in the current system, limited by our A/D converter resolution, is approximately 0.17 $\mathrm{mm}$. At a target range of $r=50 \mathrm{~cm}$ and radius $R=75 \mathrm{~mm}$, an error of $\Delta h_{\circ}=0.17 \mathrm{~mm}$ on $h_{\circ}$ corresponds to an error of $\Delta R=15.4 \mathrm{~mm}$ on $R$, which represents about a $20 \%$ error. At $r=1.0 \mathrm{~m}$, the same error on $h_{\circ}$ corresponds to $\Delta R=61.2$ $\mathrm{mm}$, representing $81 \%$ error. If $d$ is increased to $15 \mathrm{~cm}$, the error for $r=1.0 \mathrm{~m}$ is reduced to $20 \%$, and for a further increase of $d$ to $20 \mathrm{~cm}$, the error would be reduced to $11 \%$.

The fact that $\frac{\partial R}{\partial h_{\circ}}$ always takes positive values means that a measurement error $\Delta h_{\circ} \mathrm{o}+\mathrm{n} h_{\circ}$ will cause an error $\Delta R$ on $R$, having the same sign as the measurement error. This can be explained as follows. Assuming that the target has convex curvature, increasing $h_{\circ}$ while keeping $h_{r}$ and $h_{l}$ constant corresponds to an increase in the radius of curvature $R$ (or a decrease in curvature $1 / R$ ). Similarly, decreasing $h_{\circ}$ while keeping $h_{r}$ and $h_{l}$ constant corresponds to a decrease in $R$. Since the value of $\frac{\partial R}{\partial h_{0}}$ increases with range, for a fixed $\Delta h_{\circ}$, $\Delta R$ will be larger further away from the measurement system.

Since the curvature equation is symmetric with respect to $h_{r}$ and $h_{l}$, the sensitivity of curvature with respect to these two

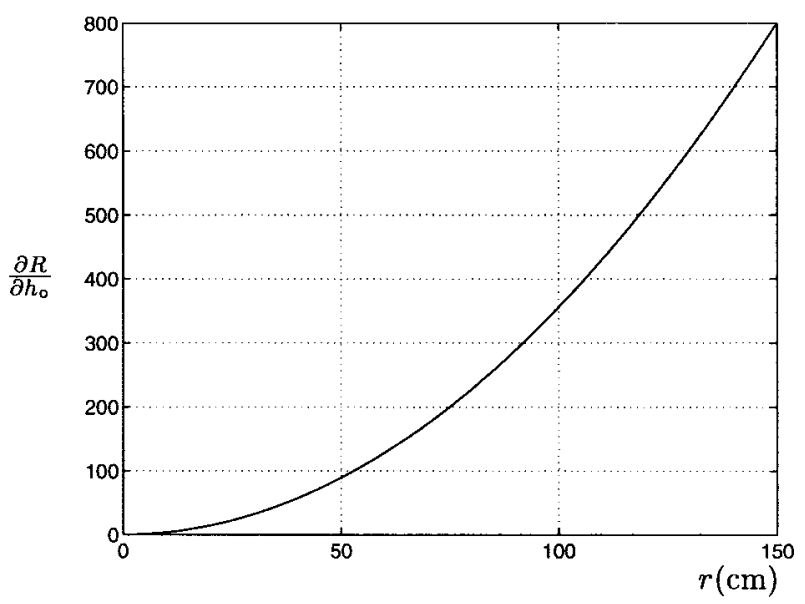

(a)

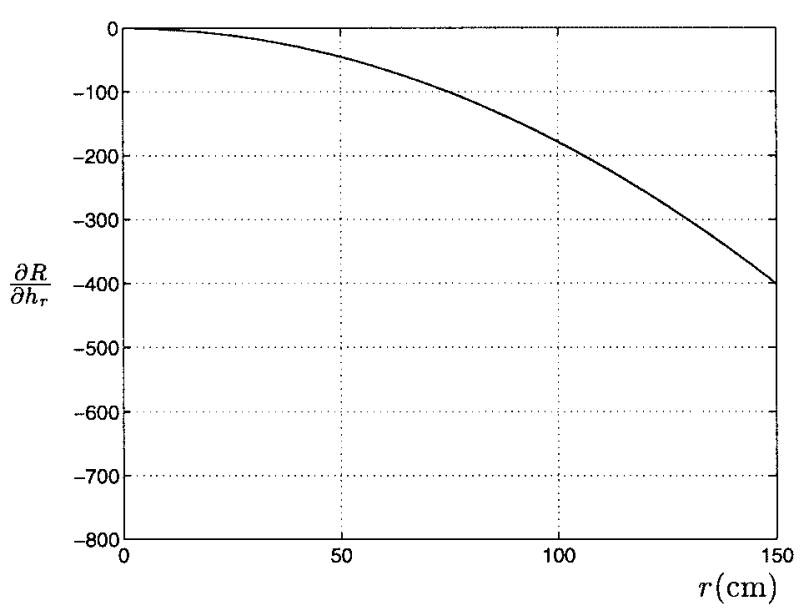

(b)

Fig. 6. Partial derivative of $R$ with respect to distance measurements (a) $h_{\circ}$ (b) $h_{r}$ or $h_{l}$, as a function of $r$. For both parts, $R=75 \mathrm{~mm}, d=7.5 \mathrm{~cm}$, $\theta=\phi=0^{\circ}$

measurements will be the same. In other words, the second and third terms in (9) are identical. In Fig. 6(b), $\frac{\partial R}{\partial h_{r}}$ has been plotted for the same parameters used in generating Fig. 6(a). Note that, in this case, $\frac{\partial R}{\partial h_{r}}$ always takes negative values and that a measurement error $\Delta h_{r}$ will cause an error $\Delta R$, having the opposite sign as the measurement error. This again can be explained by the geometry of Fig. 3. A positive (negative) error on the right and left measurements, with $h_{\circ}$ constant, causes a reduction (increase) in the radius of curvature.

Next, the sensitivity of curvature to measurement errors has been investigated for different curvature and azimuth values. In the first case, it is observed that for a fixed $h_{\circ}, \frac{\partial R}{\partial h_{\circ}}$ increases with the radius of curvature of the target, as illustrated in Fig. 7(a). In Fig. 7(b), $\theta$ is varied from $-20^{\circ}$ to $20^{\circ}$ while $d=7.5 \mathrm{~cm}$. It can be observed that the dependence of the sensitivity on $|\theta|$ is weak. Corresponding plots for $\frac{\partial R}{\partial h_{r}}$ are presented in Fig. 8(a) and (b). We observe that apart from a sign change, the general dependence of $\frac{\partial R}{\partial h_{\circ}}$ and $\frac{\partial R}{\partial h_{r}}$ on the various parameters are similar.

Fig. 7(c) illustrates the effect of varying $d$ on the sensitivity of the radius of curvature estimate to measurement errors. For $r$ between $0-1.5 \mathrm{~m}, \frac{\partial R}{\partial h_{\circ}}$ has been plotted for transducer 


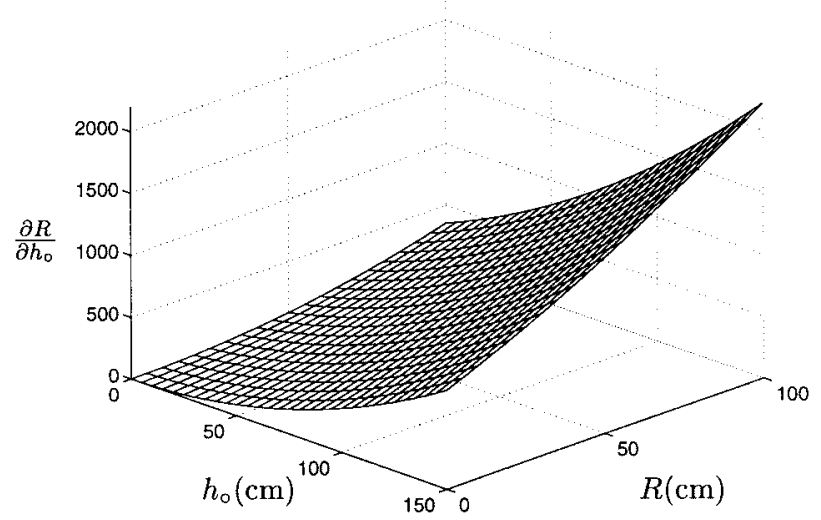

(a)

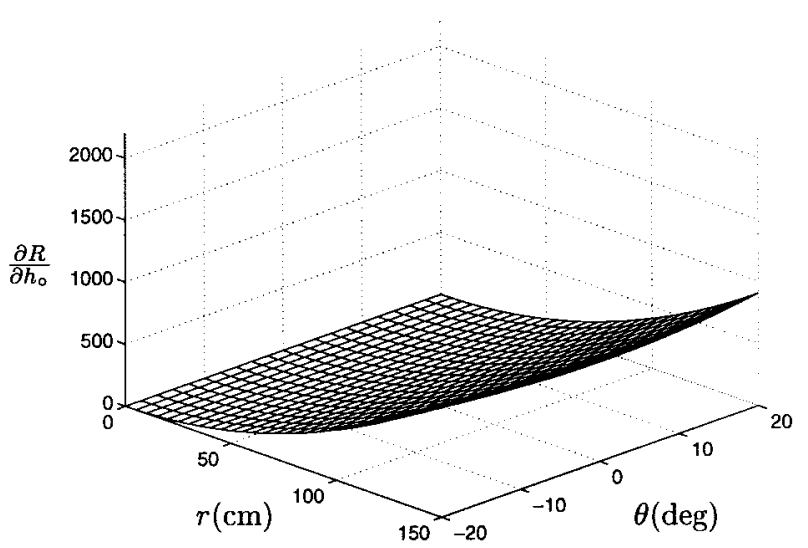

(b)

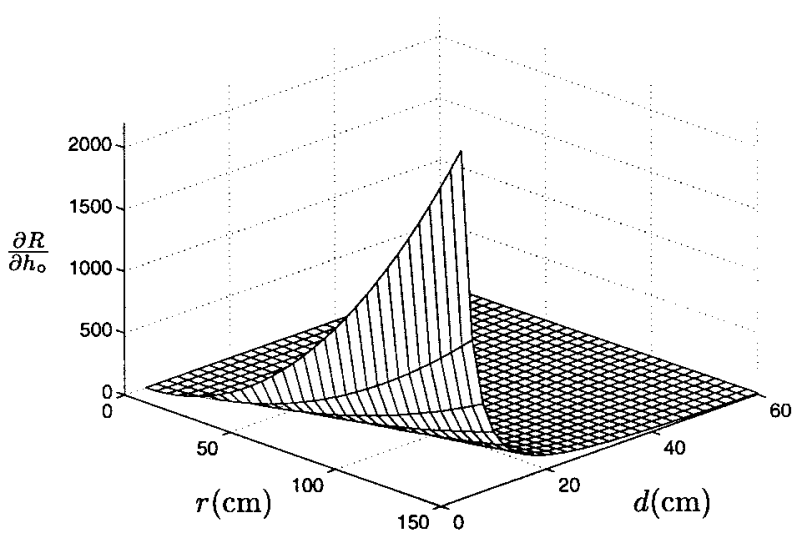

(c)

Fig. 7. Partial derivative of $R$ with respect to $h_{\circ}$ versus (a) $R$ and $h_{\circ}$ when $d=7.5 \mathrm{~cm}$ and $\theta=\phi=0^{\circ}$, (b) $\theta$ and $r$ when $d=7.5 \mathrm{~cm}, R=75 \mathrm{~mm}$ and $\phi=0^{\circ}$, (c) $d$ and $r$ when $R=75 \mathrm{~mm}, \theta=\phi=0^{\circ}$.

separations between $4.0-60 \mathrm{~cm}$. Corresponding plot for $\frac{\partial R}{\partial h_{r}}$ (or equivalently $\frac{\partial R}{\partial h_{l}}$ ) is presented in Fig. 8(c). In both figures, it is observed that the ratio $d / r$ is a significant parameter in the curvature estimation process. This ratio should be set as large as possible for better resolution. If $d / r$ is not sufficiently large, the resolution provided by the differential TOF information between the central and flanking transducers will not be large enough to estimate the curvature reliably. This is verified by the experimental results in the next section. Hence, as the operating range increases, the transducer separation must

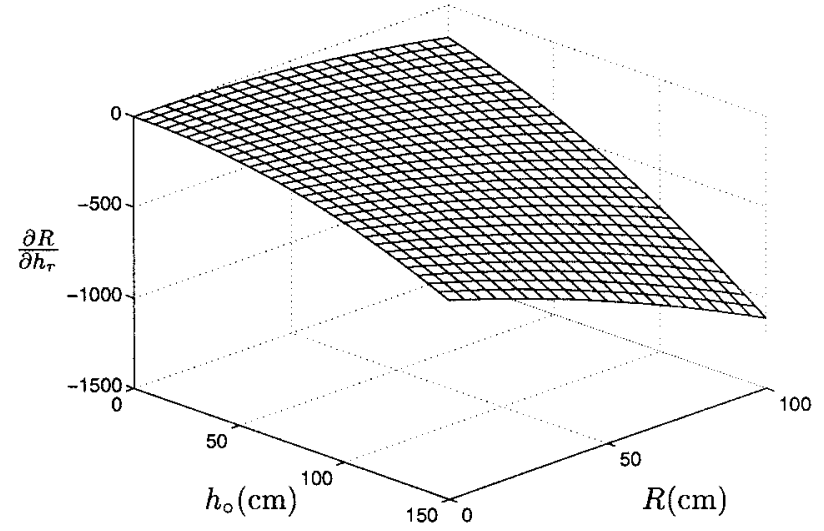

(a)

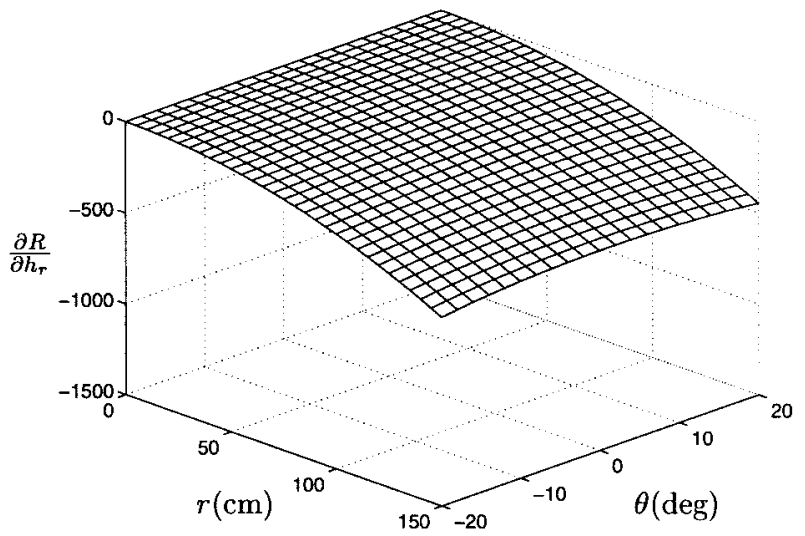

(b)

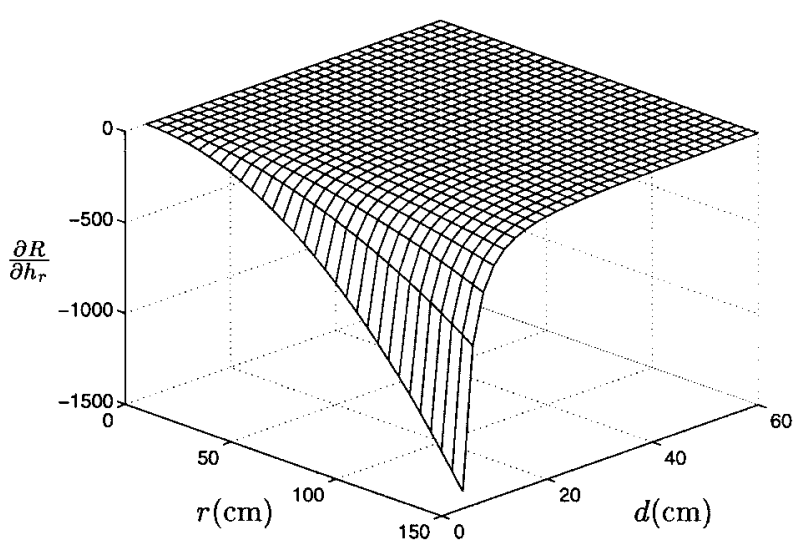

(c)

Fig. 8. Partial derivative of $R$ with respect to $h_{r}$ versus (a) $R$ and $h_{\circ}$ when $d=7.5 \mathrm{~cm}$ and $\theta=\phi=0^{\circ}$, (b) $\theta$ and $r$ when $d=7.5 \mathrm{~cm}, R=75 \mathrm{~mm}$, and $\phi=0^{\circ}$, (c) $d$ and $r$ when $R=75 \mathrm{~mm}, \theta=\phi=0^{\circ}$.

be increased accordingly to maintain the same accuracy in curvature estimation. On the other hand, there is a practical limit to how large the ratio $d / r$ can be set: The directional sensitivity patterns of the transducers are limited to a cone with half angle $\theta_{\circ}$ (in the current system, $\theta_{\circ} \cong 12^{\circ}$ [18]). If $d / r$ is too large, the sensitivity patterns of the transducers will not overlap at the location of the target so that targets nearer than $h_{\min }=\frac{d-a}{\tan \theta_{\circ}}+\frac{a^{2}}{\lambda}$ will not be detected by the system [4] as illustrated in Fig. 9. (Here, $a$ is the transducer aperture radius. For the current system with $a=20 \mathrm{~mm}$ and $\lambda=6.95 \mathrm{~mm}$, 


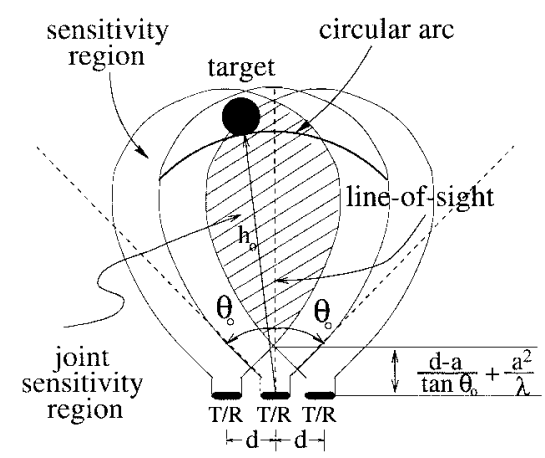

Fig. 9. Minimum distance at which a target is detectable by all three transducers is approximately $h_{\min }=\frac{d-a}{\tan \theta_{\circ}}+\frac{a^{2}}{\lambda}$ where $\theta_{\circ} \simeq 12^{\circ}$. This corresponds to the distance between the central transducer and the start of the joint sensitivity region. The system cannot detect targets outside the hatched area.

$h_{\min }=4.61 d-34.7$ in $\mathrm{mm}$.) Thus, we conclude that a sensor system which is to operate over widely varying target ranges must have the capability of adapting the transducer separation. The information provided by Figs. 7(c), 8(c), and the formula for $h_{\min }$ can be combined to formulate a rule for choosing a suitable transducer separation $d$ for a given range $r$. Thus, we can envisage a two-step curvature estimation process: The range estimate obtained in the first step is used to adjust the transducer separation $d$, allowing a more accurate curvature estimate in the second step. For example, an initial setting of $d=7.5 \mathrm{~cm}$ for a target of radius $R=75 \mathrm{~mm}$ at $r=1.0$ $\mathrm{m}$ would give $81 \%$ error when $\Delta h_{\circ}=0.17 \mathrm{~mm}$. Based on the above considerations, and applying a suitable tolerance, the initial estimate of $r$ and $R$ suggest that $d=20 \mathrm{~cm}$ would be a more suitable separation. With this value of $d$, the target surface would still be detectable by all transducers and the error would reduce to $11 \%$. However, $d$ cannot be increased much further since in this case the target would be too near to be detected by all transducers, as explained above.

Under certain circumstances, the above calculations may give a pessimistic account of the actual errors. Let us consider the hypothetical situation where all measurement errors are equal: $\Delta h_{\circ}=\Delta h_{r}=\Delta h_{l}=\Delta h$. Then, (9) becomes

$$
\Delta R=\left[\frac{\partial R}{\partial h_{\circ}}+\frac{\partial R}{\partial h_{r}}+\frac{\partial R}{\partial h_{l}}\right] \Delta h .
$$

Evaluating the partial derivatives from (2) and summing them, we obtain the remarkably simple result:

$$
\Delta R=-\Delta h .
$$

Notice that whereas the partial derivatives themselves may have very large values, they add up to -1 . The same result is also obtained from (3) or (4) when partial derivatives of these equations with respect to the corresponding measurements are evaluated and summed up. (This result is geometrically evident upon noting that an equal deviation in all measurements to the surface corresponds to an exactly opposite change in the estimate of $R$.) Therefore, any systematic source of error affecting all measurements in the same way will have negligible contribution to $\Delta R$, which will be dominated by the sources of error which affect the measurements independently. An example of a source of systematic errors is the approximately constant bias error often encountered in the detection circuitry of TOF measurement systems [2]. In certain cases, partial rather than complete cancellation may still reduce the error considerably, as with errors caused by a change in the speed of sound $c$ due to variations in the ambient conditions.

\section{B. Sensitivity to Transducer Separation}

It is also important to consider the effect of errors in setting (or measuring) the transducer separation $d$, on the radius of curvature estimation. The radius of curvature $R$ can be found from (2), (3), or (4). Suppose the transducers are positioned along the $x$ and $z$ axes of the coordinate system as before, but their locations have been set incorrectly around some nominal value of $d$. Let $d_{r}=d+\Delta d_{r}$ and $d_{l}=d+\Delta d_{l}$ be the actual, erroneously set separations of the right and left transducers. Substituting these in the first three members of (1), solving for the radius of curvature, and comparing the result with the true value of $R$, we obtain

$$
\Delta R=\frac{d\left(\Delta d_{r}+\Delta d_{l}\right)+x\left(\Delta d_{l}-\Delta d_{r}\right)+\frac{\Delta d_{r}^{2}+\Delta d_{l}^{2}}{2}}{\frac{h_{r}+h_{l}}{2}-h_{\circ}}
$$

where $x$ is the $x$-coordinate of the target center. It will be more instructive to examine the deviation in $R$ with respect to symmetric and antisymmetric components of $\Delta d_{r}$ and $\Delta d_{l}$, given by

$$
\begin{aligned}
& \Delta d_{r l}^{\mathrm{sy}} \triangleq \frac{\Delta d_{r}+\Delta d_{l}}{2} \\
& \Delta d_{r l}^{\mathrm{as}} \triangleq \frac{\Delta d_{r}-\Delta d_{l}}{2}
\end{aligned}
$$

with

$$
\begin{aligned}
\Delta d_{r} & =\Delta d_{r l}^{\mathrm{g}}-\Delta d_{r l}^{\mathrm{as}} \\
\Delta d_{l} & =\Delta d_{r l}^{\mathrm{gy}}+\Delta d_{r l}^{\mathrm{as}} .
\end{aligned}
$$

The symmetric error component $\Delta d_{r l}^{\mathrm{y}}$ corresponds to an error in the scaling of the array. On the other hand, the antisymmetric component $\Delta d_{r l}^{\text {as }}$ extends one arm and shortens the other by the same amount.

With these definitions, and neglecting second-order error terms, $\Delta R$ can be written in terms of $\Delta d_{r l}^{\mathrm{sy}}$ and $\Delta d_{r l}^{\mathrm{as}}$ as follows:

$$
\Delta R \cong \frac{d}{\frac{h_{r}+h_{l}}{2}-h_{\circ}} \Delta d_{r l}^{\mathrm{py}}+\frac{x}{\frac{h_{r}+h_{l}}{2}-h_{\circ}} \Delta d_{r l}^{\text {as }} .
$$

The sensitivity of curvature to symmetric and antisymmetric error components in $d$ has been investigated in Fig. 10 for $R=75 \mathrm{~mm}$ and $r=1.0 \mathrm{~m}$. For the case of symmetrical errors, a plot of $\frac{d}{\frac{h_{r}+h_{l}}{2}-h_{\circ}}=\frac{\partial R}{\partial d_{r l}^{\mathrm{gy}}}$ as a function of $d$ is provided in Fig. 10(a). Similarly, for antisymmetric errors, $\frac{x}{\frac{h_{r}+h_{l}}{2}-h_{\circ}}=\frac{\partial R}{\partial d_{r l}^{\text {as }}}$ is plotted in Fig. 10(b) as a function of the $x$-coordinate of the target. The $x$-coordinate of the target is varied by keeping $r=1.0 \mathrm{~m}$ and varying $\theta$ between $-20^{\circ}$ and $20^{\circ}$. Notice that $\frac{\partial R}{\partial d_{r l}^{\delta y}}$ is a sharply decreasing function of $d$, whereas $\frac{\partial R}{\partial d_{r l}^{\mathrm{as}}}$ increases roughly linearly with $x$.

The results in Fig. 10 have been extended to other parameters of interest in Figs. 11 and 12. Different curvature, azimuth, 


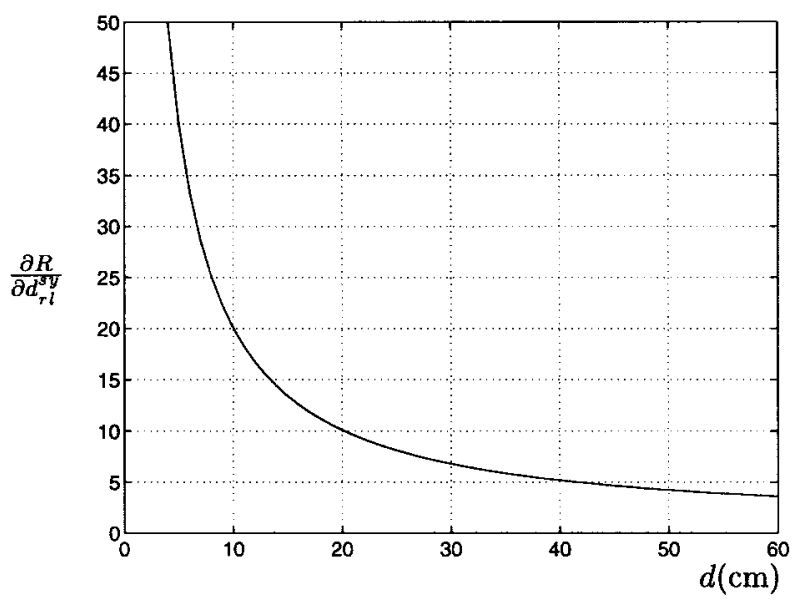

(a)

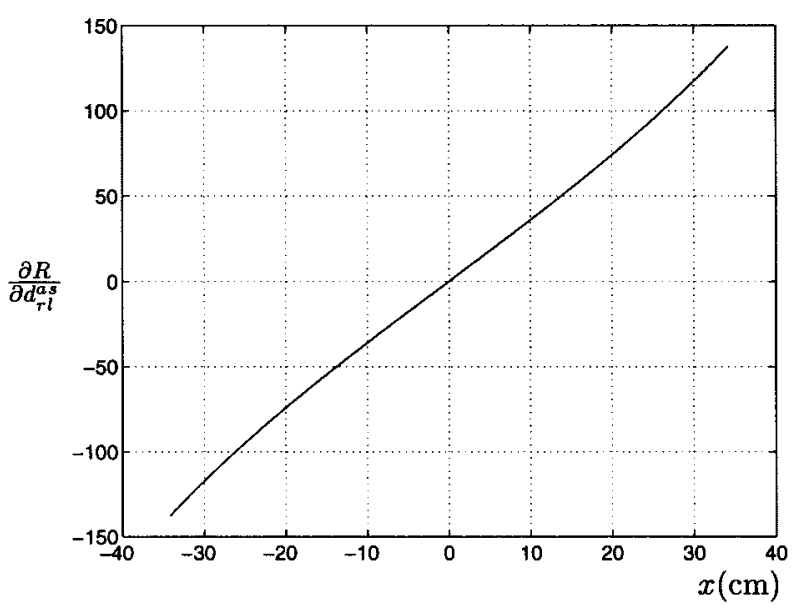

(b)

Fig. 10. Partial derivative of $R$ with respect to $d$ when the error in the separation of the right and left transducers is (a) symmetric, (b) antisymmetric. In part (a), $R=75 \mathrm{~mm}, r=1.0 \mathrm{~m}, \theta=\phi=0^{\circ}$, and in (b) $R=75 \mathrm{~mm}$, $r=1.0 \mathrm{~m}, d=7.5 \mathrm{~cm}$, and $\theta$ varies between $-20^{\circ}$ and $20^{\circ}$.

and range values have been considered. In Fig. 11(a), $\frac{\partial R}{\partial d_{r l}^{\mathrm{y}}}$ has been plotted as a function of $h_{\circ}$ and $R$. In part (b) of the same figure, $r$ has been varied between $0-1.5 \mathrm{~m}$ while $\theta$ has been varied from $-20^{\circ}$ to $20^{\circ}$, with $d=7.5 \mathrm{~cm}$. In Fig. 11(c), $\frac{\partial R}{\partial f_{r}^{y}}$ has been plotted for $d$ varying between $4-60 \mathrm{~cm}$, and $r$ between $0-1.5 \mathrm{~m}$. Once again, we observe that the dependence on $|\theta|$ is weak and the sensitivity increases rapidly for smaller values of $d / r$, so that the strategy of choosing larger values of $d$ for larger $r$ remains viable and advantageous.

The corresponding plots for the sensitivity of $R$ to the antisymmetric component of the errors on $d$, as indicated by $\frac{\partial R}{\partial d_{r l}^{\mathrm{ac}}}$, are given in Fig. 12. Whereas (a) and (c) of Figs. 11 and 12 are similar in form, the greatest difference is observed in (b) due to the appearance of the $x$-coordinate in the antisymmetric case.

Now, we turn our attention to (4) and note that an estimate of $R$ based on this equation does not depend on $d$ being accurately set or measured. It is worth examining this point more closely: Recall that (4) was derived assuming that $d_{r}=d_{l}=d_{u}=d_{d}$. Thus, if the error affecting all four transducer separations is of the same amount, (4) will give

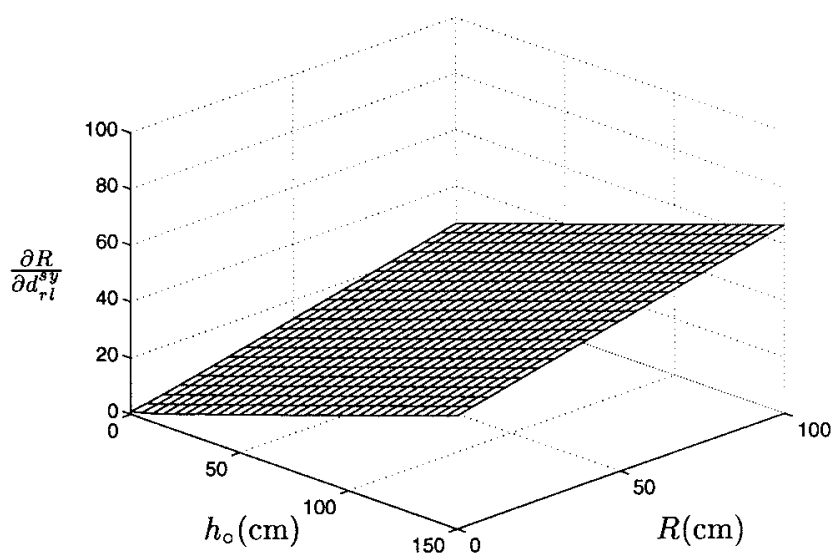

(a)

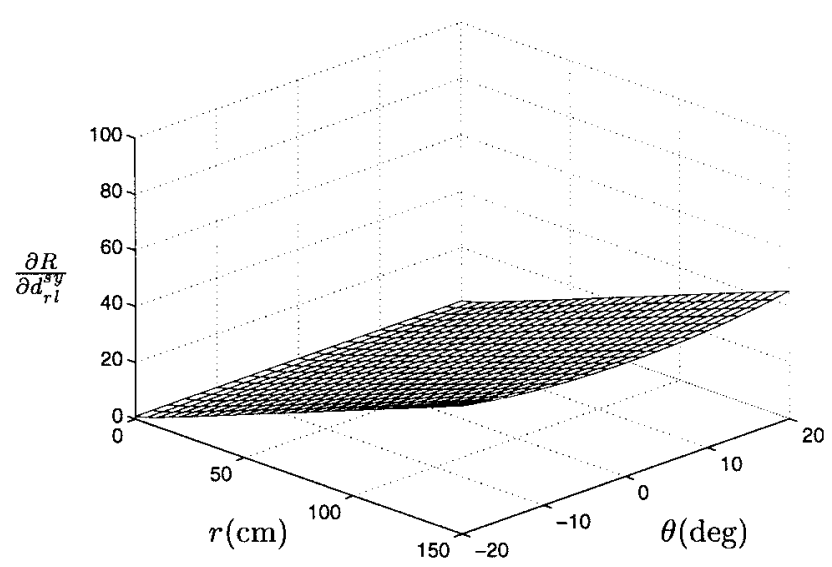

(b)

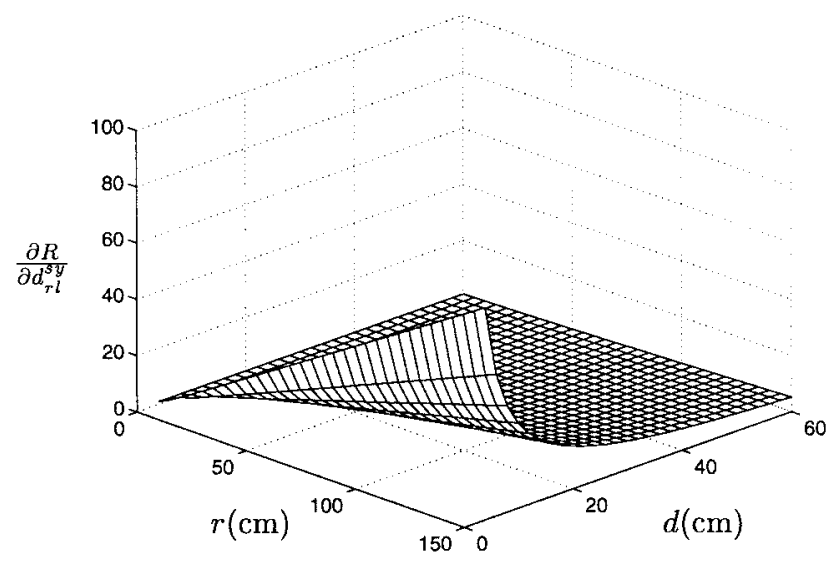

(c)

Fig. 11. Sensitivity of $R$ to symmetric error components: (a) $h_{\circ}$ and $R$ when $d=7.5 \mathrm{~cm}, \theta=\phi=0^{\circ}$, (b) $r$ and $\theta$ when $R=75 \mathrm{~mm}, d=7.5 \mathrm{~cm}$, $\phi=0^{\circ}$, (c) $r$ and $d$ when $R=75 \mathrm{~mm}, \theta=\phi=0^{\circ}$.

an estimate of $R$ independent of this error. To see what happens when errors are different for different branches, let $d_{r}=d+\Delta d_{r}, d_{l}=d+\Delta d_{l}, d_{u}=d+\Delta d_{u}$, and $d_{d}=d+\Delta d_{d}$ be the actual, erroneously set separations of the flanking transducers. Substituting these in the last four members of (1), solving for the radius of curvature and comparing the result with the true value of $R$, we obtain (16), shown at the bottom of the next page, where $z$ is the $z$-coordinate of the target 


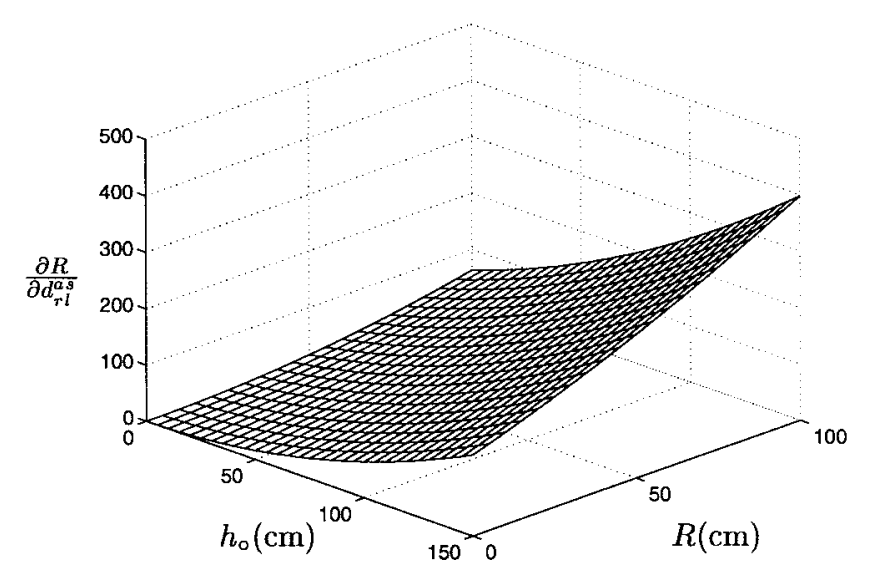

(a)

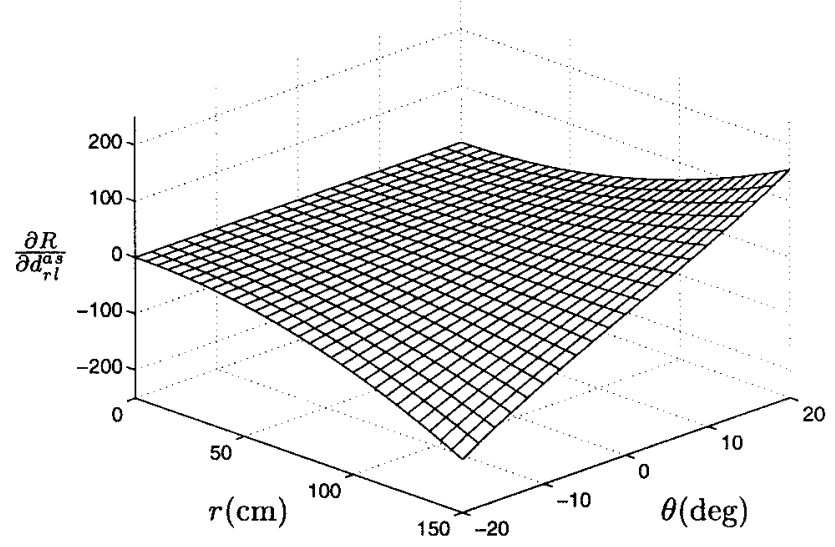

(b)

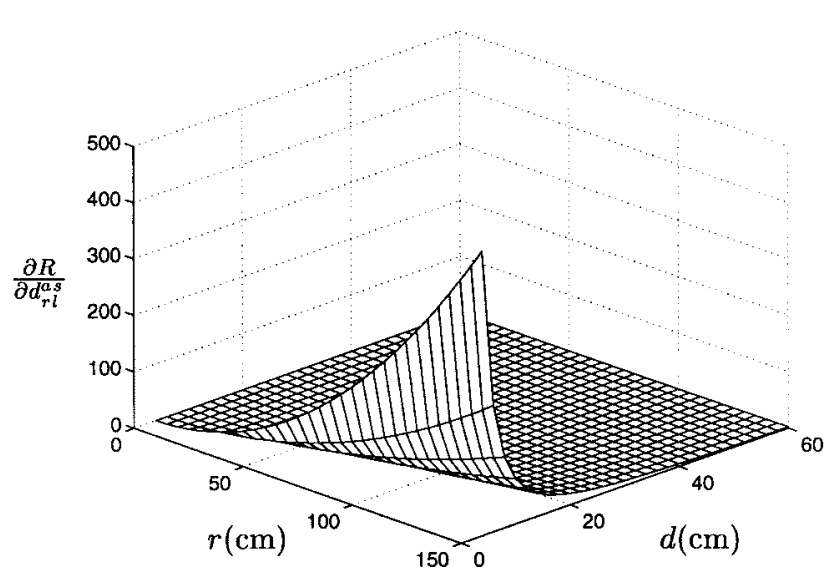

(c)

Fig. 12. Sensitivity of $R$ to antisymmetric error components: (a) $h_{\circ}$ and $R$ when $d=7.5 \mathrm{~cm}, \theta=10^{\circ}, \phi=0^{\circ}$, (b) $r$ and $\theta$ when $R=75 \mathrm{~mm}, d=7.5$ $\mathrm{cm}, \phi=0^{\circ}$, (c) $r$ and $d$ when $R=75 \mathrm{~mm}, \theta=10^{\circ}, \phi=0^{\circ}$. center. Using similar conventions as in (14), and neglecting second-order error terms

$$
\Delta R \cong \frac{-d\left(\Delta d_{r l}^{\mathrm{y}}-\Delta d_{u d}^{\mathrm{y}}\right)-x \Delta d_{r l}^{\mathrm{as}}+z \Delta d_{u d}^{\mathrm{as}}}{\frac{\left(h_{u}+h_{d}\right)}{2}-\frac{\left(h_{r}+h_{l}\right)}{2}} .
$$

As expected, the contribution to $\Delta R$ from errors affecting all branches equally, completely cancels out. Thus, in situations where the major source of error is of this type, as for instance would be the case if the error was caused by the thermal expansion of the metal chassis carrying the transducers, our estimate of $R$ will be robust to these errors. Note that this conclusion is not valid if we use (2) and (3) [see (15)]. Overall, because of the general tendency of at least some components of the errors to cancel out, we conclude that (4) is superior to (2) or (3).

As will also be confirmed experimentally in the next section, accuracy is much less of a problem with the localization parameters so that we do not present a sensitivity analysis for these parameters.

\section{EXPERIMENTAL VERIFICATION}

The analysis of the previous sections has been verified by real sonar data from cylindrical and planar targets using a 12-bit $1 \mathrm{MHz}$ PC A/D card. Echo signals were processed on an IBM-PC 486. The experiments were conducted in 2-D to allow accurate calibration. Transducer separation was kept constant at $d=7.5 \mathrm{~cm}$ except in the results presented in Table III where $d=12.0 \mathrm{~cm}$. Real distances were ascertained accurately by carrying out the whole set of experiments on large sheets of millimetric paper.

Each transducer was made to transmit and receive in sequence to avoid crosstalk and to benefit maximally from the high sampling rate of the A/D card. Starting at the transmit time, 10000 samples of each echo signal have been collected and thresholded. Since the amplitude noise standard deviation on the system is approximately $3.61 \mathrm{mV}$, the threshold level was set to $36 \mathrm{mV}$ which corresponds to ten times the noise standard deviation and $1.5 \%$ of the maximum amplitude range of the A/D card. The targets employed in this study are: cylinders with radii $25 \mathrm{~mm}, 50 \mathrm{~mm}, 75 \mathrm{~mm}$ and a planar target. The cylindrical target with radius $25 \mathrm{~mm}$ is considered a good approximation to an edge type of target formed by the intersection of two planes [12]. (Like edges, thin cylinders are highly diffractive.)

Each target's surface distance $h_{\circ}$ to the central transducer was varied between 30 to $150 \mathrm{~cm}$ at $10 \mathrm{~cm}$ intervals. At each distance, data was collected while the target was stationary at $\theta=0^{\circ}$. For the same target position, 1000 sets of measurements were taken. Each set of measurements provides a single estimate of target radius of curvature, range and azimuth. The typical differential TOF between the central

$$
\Delta R=\frac{-d\left[\left(\Delta d_{r}+\Delta d_{l}\right)-\left(\Delta d_{u}+\Delta d_{d}\right)\right]-x\left(\Delta d_{l}-\Delta d_{r}\right)+z\left(\Delta d_{d}+\Delta d_{u}\right)-\frac{\left(\Delta d_{r}^{2}+\Delta d_{l}^{2}\right)}{2}-\frac{\left(\Delta d_{u}^{2}+\Delta d_{d}^{2}\right)}{2}}{\left(h_{u}+h_{d}\right)-\left(h_{r}+h_{l}\right)}
$$


TABLE I

EXPERIMENTAL RESULTS FOR A CYLINDER OF $R=75 \mathrm{~mm}$ WHEN $d=7.5 \mathrm{~cm}$

\begin{tabular}{c||c||c||c||c||c||c}
\hline$h_{\circ}$ & $\begin{array}{c}E\left\{\hat{h}_{\circ}\right\} \\
(\mathrm{mm})\end{array}$ & $\begin{array}{c}\sigma_{\hat{h}_{\circ}} \\
(\mathrm{mm})\end{array}$ & $\begin{array}{c}E\{\hat{\theta}\} \\
(\mathrm{mm})\end{array}$ & $\begin{array}{c}\sigma_{\hat{\theta}} \\
(\mathrm{deg})\end{array}$ & $\begin{array}{c}E\{\hat{R}\} \\
(\mathrm{deg})\end{array}$ & $\begin{array}{c}\sigma_{\hat{R}} \\
(\mathrm{~mm})\end{array}$ \\
\hline 300 & 299.93 & 0.14 & 0.01 & 0.08 & 73.4 & 7.8 \\
\hline 400 & 400.00 & 0.14 & 0.02 & 0.07 & 78.8 & 11.8 \\
\hline 500 & 500.15 & 0.14 & -0.04 & 0.07 & 75.1 & 17.1 \\
\hline 600 & 600.02 & 0.14 & -0.04 & 0.07 & 75.7 & 24.4 \\
\hline 700 & 700.10 & 0.18 & 0.01 & 0.07 & 81.7 & 32.0 \\
\hline 800 & 800.15 & 0.14 & 0.02 & 0.07 & 80.3 & 38.8 \\
\hline 900 & 900.42 & 0.14 & 0.07 & 0.07 & 71.1 & 50.3 \\
\hline 1000 & 1000.24 & 0.16 & -0.02 & 0.07 & 76.3 & 60.2 \\
\hline 1100 & 1100.23 & 0.14 & -0.03 & 0.07 & 75.6 & 70.9 \\
\hline 1200 & 1200.07 & 0.14 & 0.02 & 0.07 & 80.5 & 84.5 \\
\hline 1300 & 1300.19 & 0.14 & 0.00 & 0.07 & 78.7 & 95.5 \\
\hline 1400 & 1400.37 & 0.14 & 0.01 & 0.07 & 80.1 & 124.4 \\
\hline 1500 & 1500.22 & 0.15 & 0.01 & 0.07 & 69.2 & 118.2 \\
\hline
\end{tabular}

TABLE II

EXPERIMENTAL Results FOR A CyLINDER OF $R=50 \mathrm{~mm}$ When $d=7.5 \mathrm{~cm}$

\begin{tabular}{c||c||c||c||c||c||c}
\hline$h_{\circ}$ & $\begin{array}{c}E\left\{\hat{h}_{\circ}\right\} \\
(\mathrm{mm})\end{array}$ & $\begin{array}{c}\sigma_{\hat{h}_{\circ}} \\
(\mathrm{mm})\end{array}$ & $\begin{array}{c}E\{\hat{\theta}\} \\
(\mathrm{mm})\end{array}$ & $\begin{array}{c}\sigma_{\hat{\theta}} \\
(\mathrm{deg})\end{array}$ & $\begin{array}{c}E\{\hat{R}\} \\
(\mathrm{deg})\end{array}$ & $\begin{array}{c}\sigma_{\hat{R}} \\
(\mathrm{~mm})\end{array}$ \\
\hline \hline 300 & 300.00 & 0.14 & -0.03 & 0.09 & 48.3 & 6.8 \\
\hline 400 & 399.79 & 0.14 & -0.02 & 0.07 & 51.1 & 10.1 \\
\hline 500 & 500.05 & 0.16 & -0.04 & 0.07 & 49.2 & 15.2 \\
\hline 600 & 600.59 & 0.17 & 0.04 & 0.07 & 52.3 & 22.4 \\
\hline 700 & 699.98 & 0.15 & 0.01 & 0.07 & 45.0 & 27.6 \\
\hline 800 & 799.87 & 0.14 & -0.03 & 0.07 & 44.6 & 35.0 \\
\hline 900 & 900.40 & 0.15 & 0.05 & 0.07 & 50.6 & 45.4 \\
\hline 1000 & 1000.39 & 0.14 & 0.03 & 0.07 & 59.9 & 57.4 \\
\hline 1100 & 1100.17 & 0.15 & -0.10 & 0.07 & 53.8 & 64.7 \\
\hline 1200 & 1200.14 & 0.14 & 0.01 & 0.07 & 48.1 & 83.7 \\
\hline 1300 & 1300.15 & 0.14 & -0.05 & 0.07 & 63.7 & 91.4 \\
\hline 1400 & 1400.63 & 0.15 & -0.08 & 0.07 & 40.9 & 122.5 \\
\hline 1500 & 1500.18 & 0.15 & 0.01 & 0.07 & 46.6 & 125.5 \\
\hline \hline 50
\end{tabular}

TABLE III

EXPERIMENTAL RESULTS FOR A CYLINDER OF $R=50 \mathrm{~mm}$ wITH $d=12.0 \mathrm{~cm}$

\begin{tabular}{c||c||c||c||c||c||c}
\hline \hline$h_{\circ}$ & $E\left\{\hat{h}_{\circ}\right\}$ & $\sigma_{\hat{h}_{\circ}}$ & $E\{\hat{\theta}\}$ & $\sigma_{\hat{\theta}}$ & $E\{\hat{R}\}$ & $\sigma_{\hat{R}}$ \\
$(\mathrm{~mm})$ & $(\mathrm{mm})$ & $(\mathrm{mm})$ & $(\mathrm{deg})$ & $(\mathrm{deg})$ & $(\mathrm{mm})$ & $(\mathrm{mm})$ \\
\hline \hline 300 & - & - & - & - & - & - \\
\hline 400 & - & - & - & - & - & - \\
\hline 500 & 499.75 & 0.14 & -0.07 & 0.05 & 48.2 & 6.2 \\
\hline 600 & 599.97 & 0.17 & -0.08 & 0.05 & 49.5 & 9.0 \\
\hline 700 & 700.10 & 0.15 & 0.00 & 0.05 & 47.2 & 11.4 \\
\hline 800 & 799.93 & 0.15 & 0.02 & 0.05 & 48.6 & 15.2 \\
\hline 900 & 900.20 & 0.16 & 0.00 & 0.05 & 52.2 & 19.1 \\
\hline 1000 & 999.77 & 0.15 & -0.02 & 0.05 & 46.8 & 22.5 \\
\hline 1100 & 1100.43 & 0.16 & -0.03 & 0.05 & 55.4 & 27.5 \\
\hline 1200 & 1200.11 & 0.16 & -0.03 & 0.05 & 45.1 & 31.7 \\
\hline 1300 & 1300.34 & 0.15 & -0.02 & 0.04 & 48.5 & 38.6 \\
\hline 1400 & 1400.26 & 0.17 & -0.02 & 0.05 & 47.5 & 43.5 \\
\hline 1500 & 1500.37 & 0.14 & -0.07 & 0.04 & 49.4 & 46.8 \\
\hline
\end{tabular}

transducer and the right/left transducers varies between $0-10$ $\mathrm{mm}$ depending on the target curvature and distance, for $d=7.5 \mathrm{~cm}$. As the range of the target increases, the differential signal becomes less reliable to extract the curvature information.

The means and standard deviations of $h_{\circ}, \theta$ and $R$ (or $R^{-1}$ ) of each type of target considered are computed and tabulated in Tables I-V. In all of the tables, results for $h_{\circ}$ and $R$ are tabulated individually instead of $r$ itself, which is the sum of these two components. In Table III, results for $h_{\circ}=300$ $\mathrm{mm}$ and $h_{\circ}=400 \mathrm{~mm}$ have not been presented since a very thin cylinder at $\theta=0^{\circ}$ is not detectable by the right and left transducers at the transducer separation of $d=12.0 \mathrm{~cm}$. For the same reason, results for $h_{\circ}=300 \mathrm{~mm}$ in Table IV are excluded when $d$ was set equal to $7.5 \mathrm{~cm}$. From the results, it can be observed that the $h_{\circ}$ and $\theta$ estimates are quite accurate: For a stationary target, the typical standard deviation of the $h_{\circ}$ measurement is $0.17 \mathrm{~mm}$. The typical standard deviation of azimuth estimate is $0.08^{\circ}$. Error on $h_{\circ}$ and $\theta$ are relatively constant as the distance of the target is varied between 30-150 $\mathrm{cm}$. However, for the curvature, typical error is around 7-9 $\mathrm{mm}$ at $30 \mathrm{~cm}$, but keeps increasing with range for a fixed transducer separation. This is due to the reduction in the $d / r$ ratio which provides poorer resolution in estimating curvature. To estimate curvature of a cylindrical target reliably, it is necessary to increase the transducer separation as the range is increased as seen in Fig. 6(a). To illustrate the effect of transducer separation, results for the maximum allowed separation in our system $(d=12.0 \mathrm{~cm})$ are included in Table III. Compared 
TABLE IV

EXPERIMENTAL RESUlts FOR A CYLINDER OF $R=25 \mathrm{~mm}$ When $d=7.5 \mathrm{~cm}$

\begin{tabular}{|c|c|c|c|c|c|c|}
\hline $\begin{array}{c}h_{\circ} \\
(\mathrm{mm})\end{array}$ & $\begin{array}{l}E\left\{\hat{h}_{\circ}\right\} \\
(\mathrm{mm})\end{array}$ & $\begin{array}{c}\sigma_{\hat{h}_{\circ}} \\
(\mathrm{mm})\end{array}$ & $\begin{array}{l}E\{\hat{\theta}\} \\
(\mathrm{deg})\end{array}$ & $\begin{array}{c}\sigma_{\hat{\theta}} \\
(\mathrm{deg})\end{array}$ & $\begin{array}{l}E\{\hat{R}\} \\
(\mathrm{mm})\end{array}$ & $\begin{array}{c}\sigma_{\hat{R}} \\
(\mathrm{~mm})\end{array}$ \\
\hline 300 & - & - & - & - & - & - \\
\hline 400 & 400.06 & 0.16 & 0.02 & 0.08 & 21.1 & 9.1 \\
\hline 500 & 500.11 & 0.14 & -0.03 & 0.07 & 23.6 & 14.0 \\
\hline 600 & 599.99 & 0.15 & 0.04 & 0.07 & 28.6 & 20.1 \\
\hline 700 & 699.99 & 0.15 & -0.05 & 0.07 & 26.2 & 26.0 \\
\hline 800 & 799.77 & 0.16 & 0.02 & 0.07 & 26.5 & 34.2 \\
\hline 900 & 899.91 & 0.20 & 0.02 & 0.07 & 30.4 & 43.0 \\
\hline 1000 & 999.44 & 0.24 & 0.03 & 0.08 & 29.9 & 57.5 \\
\hline 1100 & 1100.18 & 0.17 & 0.02 & 0.07 & 28.5 & 64.3 \\
\hline 1200 & 1200.44 & 0.16 & -0.04 & 0.07 & 23.5 & 78.0 \\
\hline 1300 & 1300.31 & 0.18 & -0.04 & 0.07 & 23.7 & 91.5 \\
\hline 1400 & 1400.01 & 0.15 & 0.00 & 0.07 & 23.5 & 98.6 \\
\hline 1500 & 1499.39 & 0.25 & 0.03 & 0.08 & 23.0 & 139.2 \\
\hline
\end{tabular}

to Table II where $d$ was $7.5 \mathrm{~cm}$, we observe that errors in the radius of curvature estimate are approximately reduced by $60 \%$.

In the lower right corner of Table II, $E\{R\}$ is within $10 \%$ of the true value of $R$, but $\sigma_{R} \cong 2.5 E\{R\}$. Since both $E\{R\}$ and $\sigma_{R}$ were estimated over 1000 realizations, the error in the calculated value of $E\{R\}$ is expected to be $\frac{\sigma_{r}}{\sqrt{1000}}$. Therefore, we obtain $\frac{\sigma_{r}}{\sqrt{1000}}=\frac{2.5 E\{R\}}{\sqrt{1000}}=0.08 E\{R\}$. This is indeed in agreement with the fact that $E\{R\}$ is obtained within $10 \%$ of its correct value. This case represents one of the worst considered in the experiments.

In Table $\mathrm{V}$, results for a planar target $(R=\infty)$ are illustrated. When a set of measurements indicates that $R$ is indistinguishable from infinity (i.e., the denominator in (2) is experimentally indistinguishable from zero) the planar target formulas derived in Section III-C are used instead of (2) and (5). Since in this case the denominator of (2) is very small, it is preferable to deal with curvature $R^{-1}$ rather than the radius of curvature $R$.

In Table VI, results for the cylinder with $R=25 \mathrm{~mm}$ are provided for $\theta=0^{\circ}, 3^{\circ}, 5^{\circ}, 8^{\circ}$. It is observed that the accuracy of range and azimuth estimates do not change significantly as compared to the case when the target is along the line-of-sight $\left(\theta=0^{\circ}\right)$. For larger values of $\theta$ than considered in the table, it is not possible to estimate the curvature since the target will be outside the sensitivity pattern of either the right or the left transducer and there will not be a sufficient number of measurements.
TABLE V

EXPERImental Results For a Planar TARget of $R=\infty$ When $d=7.5 \mathrm{~cm}$

\begin{tabular}{c||c||c||c||c||c||c}
\hline \hline$h_{\circ}$ & $\begin{array}{c}E\left\{\hat{h}_{\circ}\right\} \\
(\mathrm{mm})\end{array}$ & $\begin{array}{c}\sigma_{\hat{h}_{\circ}} \\
(\mathrm{mm})\end{array}$ & $\begin{array}{c}E\{\hat{\theta}\} \\
(\mathrm{mm})\end{array}$ & $\begin{array}{c}\sigma_{\hat{\theta}} \\
(\mathrm{deg})\end{array}$ & $\begin{array}{c}E\left\{\hat{R}^{-1}\right\} \\
\left(\mathrm{mm}^{-1}\right)\end{array}$ & $\begin{array}{c}\sigma_{\hat{R}^{-1}} \\
\left(\mathrm{~mm}^{-1}\right)\end{array}$ \\
\hline 300 & 299.88 & 0.14 & -0.02 & 0.08 & $-9.1 \times 10^{-5}$ & $5.6 \times 10^{-5}$ \\
\hline 400 & 399.94 & 0.14 & 0.01 & 0.08 & $2.3 \times 10^{-5}$ & $6.1 \times 10^{-5}$ \\
\hline 500 & 500.09 & 0.15 & -0.04 & 0.08 & $5.6 \times 10^{-5}$ & $6.4 \times 10^{-5}$ \\
\hline 600 & 600.11 & 0.16 & -0.01 & 0.07 & $1.5 \times 10^{-5}$ & $6.2 \times 10^{-5}$ \\
\hline 700 & 699.98 & 0.16 & -0.02 & 0.08 & $3.9 \times 10^{-5}$ & $6.5 \times 10^{-5}$ \\
\hline 800 & 800.17 & 0.16 & 0.10 & 0.08 & $5.1 \times 10^{-5}$ & $6.5 \times 10^{-5}$ \\
\hline 900 & 899.88 & 0.16 & 0.10 & 0.08 & $4.7 \times 10^{-5}$ & $6.5 \times 10^{-5}$ \\
\hline 1000 & 1000.07 & 0.15 & 0.03 & 0.08 & $7.7 \times 10^{-5}$ & $7.3 \times 10^{-5}$ \\
\hline 1100 & 1100.18 & 0.16 & 0.04 & 0.08 & $7.7 \times 10^{-5}$ & $7.2 \times 10^{-5}$ \\
\hline 1200 & 1199.92 & 0.17 & -0.04 & 0.08 & $9.2 \times 10^{-5}$ & $7.9 \times 10^{-5}$ \\
\hline 1300 & 1300.00 & 0.18 & -0.05 & 0.08 & $7.0 \times 10^{-5}$ & $7.5 \times 10^{-5}$ \\
\hline 1400 & 1399.56 & 0.16 & 0.00 & 0.07 & $4.1 \times 10^{-5}$ & $6.9 \times 10^{-5}$ \\
\hline 1500 & 1500.34 & 0.20 & 0.01 & 0.08 & $8.9 \times 10^{-5}$ & $7.7 \times 10^{-5}$ \\
\hline
\end{tabular}

TABLE VI

EXPERIMENTAL RESULTS FOR A CYLINDER WITH $R=25 \mathrm{~mm}$ FOR VARYING $\theta$ WHEN $d=7.5 \mathrm{~cm}$

\begin{tabular}{|c|c|c|c|c|c|c|}
\hline $\begin{array}{c}\theta \\
(\operatorname{deg})\end{array}$ & $\begin{array}{r}E\left\{\hat{h}_{\circ}\right\} \\
(\mathrm{mm})\end{array}$ & $\begin{array}{c}\sigma_{\hat{h}_{\circ}} \\
(\mathrm{mm})\end{array}$ & $\begin{array}{r}E\{\hat{\theta}\} \\
(\operatorname{deg})\end{array}$ & $\begin{array}{c}\sigma_{\hat{\theta}} \\
(\operatorname{deg})\end{array}$ & $\begin{array}{r}E\{\hat{R}\} \\
(\mathrm{mm})\end{array}$ & $\begin{array}{c}\sigma_{\hat{R}} \\
(\mathrm{~mm})\end{array}$ \\
\hline 0 & 1000.26 & 0.16 & -0.04 & 0.07 & 22.7 & 56.3 \\
\hline 3 & 999.46 & 0.15 & 2.73 & 0.08 & 24.6 & 55.2 \\
\hline 5 & 1000.67 & 0.15 & 4.98 & 0.07 & 25.6 & 53.5 \\
\hline 8 & 1000.05 & 0.16 & 7.53 & 0.07 & 27.3 & 43.8 \\
\hline
\end{tabular}

The returned signal intensity from a cylindrical target is $I_{\text {ret, }, \text { cy }} \cong \frac{\sigma_{\mathrm{cy}}}{2 \pi r} I$, and from a sphere is $I_{\mathrm{ret}, \mathrm{sp}} \cong \frac{\sigma_{\mathrm{sp}}}{4 \pi r^{2}} I$, where $I=f(\theta, \phi) / r^{2}$ is the intensity of the incident wave, and $\sigma_{\mathrm{cy}}$ is the scattering cross section of the cylinder per unit length, whereas $\sigma_{\mathrm{sp}}$ is the scattering cross section of the sphere. For a rigid cylinder of radius $R$, geometrical (or ray) acoustics (i.e., the limit $k R \gg 1$ ) provides an approximate cross section of $4 R$ per unit length of the cylinder, where $k=\frac{2 \pi}{\lambda}$ is the acoustic wavenumber [16]. For the cylinders used in the experiments, $k R$ ranges between 22 and 68 so that $k R \gg 1$ is valid. For a rigid sphere of radius $R$, the approximate scattering cross section is $2 \pi R^{2}$ for $k R \gg 1$ [16]. Substituting in for the scattering cross sections, we obtain: $I_{\text {ret, cy }}=\frac{2 R}{\pi r} I$ and $I_{\text {ret, } \mathrm{sp}}=\frac{R^{2}}{2 r^{2}} I$. For example, considering a sphere of radius 
$7.5 \mathrm{~cm}$ located at $r=50 \mathrm{~cm}$, the measured signal amplitude $\left(\propto \sqrt{I_{\text {ret }}}\right)$ from the sphere would be approximately a third of that from the cylinder. However, such a reduction in the signal amplitude does not result in a proportionate decrease in TOF measurement accuracy, as long as the target is within the sensitivity region and the amplitude remains above the threshold [5]. Thus, the main difference between a cylinder and a sphere of comparable radius is not accuracy but the size of the region in which they can be detected, which is smaller for the sphere.

\section{CONCLUSION}

A sensing device capable of estimating the location and radius of curvature of spherical and cylindrical targets has been described. Two limiting cases are of special interest: the point (in 3-D) or line (in 2-D) target and the planar target. Sensitivity analysis of the curvature estimate with respect to various types of measurement errors and some system parameters is provided. Analytical results are verified by real sonar data from cylindrical and planar targets. Typical accuracies in range and azimuth are $0.17 \mathrm{~mm}$ and $0.1^{\circ}$ respectively. Accuracy of the curvature estimate depends on the target type and system parameters such as transducer separation and operating range. For reliable curvature estimation, it is necessary to increase the transducer separation as the range is increased. The transducer separation in our system is relatively limited and not capable of real-time dynamic adaptation.

Current and future work will focus on improving the robustness of the radius of curvature estimation by using recursive digital filtering techniques. This will reduce the variance of the estimates and thus improve their reliability. More efficient firing techniques involving cross firing patterns will be considered to reduce the data acquisition time. In addition to TOF information, incorporation of amplitude information or the shape of the complete echo waveform in the current system will provide additional information about the location and curvature of the sonified target. Since the time this paper was submitted, an alternative approach based on morphological processing, that can handle surfaces with spatially varying curvature which may become both concave and convex, has also been developed [6].

\section{APPENDIX}

A solution using only four measurements (i.e., the last four equations in equation set 1 ) has been obtained by Mathematica
[20] in Cartesian coordinates as follows:

$$
\begin{aligned}
y= & \frac{1}{2 \sqrt{2}}\left(\left(-\frac{1}{d^{2}\left(h_{u}+h_{d}-h_{r}-h_{l}\right)^{2}}\left(2 h_{l}^{4} h_{r}^{2}-4 h_{l}^{3} h_{r}^{3}\right.\right.\right. \\
& +2 h_{l}^{2} h_{r}^{4}-2 h_{l}^{4} h_{r} h_{u}+2 h_{l}^{3} h_{r}^{2} h_{u}+2 h_{l}^{2} h_{r}^{3} h_{u}-2 h_{l} h_{r}^{4} h_{u} \\
& +h_{l}^{4} h_{u}^{2}+2 h_{l}^{3} h_{r} h_{u}^{2}-4 h_{l}^{2} h_{r}^{2} h_{u}^{2}+2 h_{l} h_{r}^{3} h_{u}^{2}+h_{r}^{4} h_{u}^{2} \\
& -2 h_{l}^{3} h_{u}^{3}-2 h_{r}^{3} h_{u}^{3}+h_{l}^{2} h_{u}^{4}+h_{r}^{2} h_{u}^{4} \\
& +8 d^{4}\left(h_{d}-h_{l}-h_{r}+h_{u}\right)^{2} \\
& +h_{d}^{4}\left(h_{l}^{2}+h_{r}^{2}-2 h_{l} h_{u}-2 h_{r} h_{u}+2 h_{u}^{2}\right) \\
& -2 h_{d}^{3}\left(h_{l}^{3}+h_{r}^{3}-h_{l}^{2} h_{u}-h_{r}^{2} h_{u}-h_{l} h_{u}^{2}-h_{r} h_{u}^{2}+2 h_{u}^{3}\right) \\
& +h_{d}^{2}\left(h_{l}^{4}+2 h_{l}^{3} h_{r}-4 h_{l}^{2} h_{r}^{2}+2 h_{l} h_{r}^{3}+h_{r}^{4}\right. \\
& +2 h_{l}^{2} h_{r} h_{u}+2 h_{l} h_{r}^{2} h_{u}-4 h_{l}^{2} h_{u}^{2}-4 h_{l} h_{r} h_{u}^{2}-4 h_{r}^{2} h_{u}^{2} \\
& \left.+2 h_{l} h_{u}^{3}+2 h_{r} h_{u}^{3}+2 h_{u}^{4}\right) \\
& -2 h_{d}\left(h_{l}^{4} h_{r}-h_{l}^{3} h_{r}^{2}-h_{l}^{2} h_{r}^{3}+h_{l} h_{r}^{4}+2 h_{l}^{2} h_{r}^{2} h_{u}\right. \\
& \left.-h_{l}^{2} h_{r} h_{u}^{2}-h_{l} h_{r}^{2} h_{u}^{2}-h_{l}^{2} h_{u}^{3}-h_{r}^{2} h_{u}^{3}+h_{l} h_{u}^{4}+h_{r} h_{u}^{4}\right) \\
& -2 d^{2}\left(h_{d}^{4}+h_{l}^{4}+2 h_{l}^{2} h_{r}^{2}+h_{r}^{4}-2 h_{d}^{3}\left(h_{l}+h_{r}\right)\right. \\
& -2 h_{l}^{3} h_{u}-2 h_{l}^{2} h_{r} h_{u}-2 h_{l} h_{r}^{2} h_{u}-2 h_{r}^{3} h_{u}+2 h_{l}^{2} h_{u}^{2} \\
& +4 h_{l} h_{r} h_{u}^{2}+2 h_{r}^{2} h_{u}^{2}-2 h_{l} h_{u}^{3}-2 h_{r} h_{u}^{3}+h_{u}^{4} \\
& +2 h_{d}^{2}\left(h_{l}^{2}+2 h_{l} h_{r}+h_{r}^{2}-h_{l} h_{u}-h_{r} h_{u}+h_{u}^{2}\right) \\
& -2 h_{d}\left(h_{l}^{3}+h_{l}^{2} h_{r}+h_{l} h_{r}^{2}+h_{r}^{3}-2 h_{l}^{2} h_{u}-2 h_{r}^{2} h_{u}\right. \\
& \left.\left.\left.\left.+h_{l} h_{u}^{2}+h_{r} h_{u}^{2}\right)\right)\right)\right) \\
&
\end{aligned}
$$

and in (19)-(21), shown at the bottom of the page.

\section{ACKNOWLEDGMENT}

The author would like to thank Dr. M. Siegel, Carnegie Mellon University, and Dr. V, Grinberg of Synopsys Corporation, Mountain View, CA, for their input and many useful discussions, and the anonymous reviewers for their comments and suggestions.

\section{REFERENCES}

[1] B. Ayrulu and B. Barshan, "Identification of target primitives with multiple decision-making sonars using evidential reasoning," Int. J. Robot. Res., vol. 17, no. 6, pp. 598-623, 1998.

[2] B. Barshan and B. Ayrulu, "Performance comparison of four methods of time-of-flight estimation for sonar waveforms," Electron. Lett., vol. 34, pp. 1616-1617, Aug. 1998.

[3] B. Barshan and O. Arr1kan, "Performance analysis of two linear array processing algorithms for point-obstacle localization," in Proc. SPIE

$$
\begin{aligned}
& x=\frac{\left(h_{l}-h_{r}\right)\left(h_{d}^{2}+2 h_{l} h_{r}-h_{d}\left(h_{l}+h_{r}\right)-h_{l} h_{u}-h_{r} h_{u}+h_{u}^{2}\right)}{4 d\left(h_{r}+h_{l}-h_{u}-h_{d}\right)} \\
& z=\frac{h_{d}^{2}\left(h_{l}+h_{r}-2 h_{u}\right)-h_{d}\left(h_{l}^{2}+h_{r}^{2}-2 h_{u}^{2}\right)-h_{u}\left[-h_{l}^{2}+h_{l} h_{u}+h_{r}\left(h_{u}-h_{r}\right)\right]}{4 d\left(h_{r}+h_{l}-h_{u}-h_{d}\right)} \\
& R=\frac{\left(h_{u}^{2}+h_{d}^{2}\right)-\left(h_{r}^{2}+h_{l}^{2}\right)}{2\left(h_{r}+h_{l}-h_{u}-h_{d}\right)} .
\end{aligned}
$$


Signal Data Processing Small Targets, O. E. Drummond, Ed. San Diego, CA, July 11-13, 1995, vol. 2561, pp. 533-544.

[4] B. Barshan and R. Kuc, "Differentiating sonar reflections from corners and planes by employing an intelligent sensor," IEEE Trans. Pattern Anal. Machine Intell., vol. 12, pp. 560-569, June 1990.

[5] "A bat-like sonar system for obstacle localization," IEEE Trans. Syst., Man, Cybern, vol. 22, pp. 636-646, July/Aug. 1992

[6] D. Başkent and B. Barshan, "Surface profile determination from multiple sonar data using morphological processing," Int. J. Robot. Res., vol. 18, pp. 788-808, Aug. 1999.

[7] Ö. Bozma and R. Kuc, "Characterizing pulses reflected from rough surfaces using ultrasound,” J. Acoust. Soc. Amer., vol. 89, pp. 2519-2531, June 1991.

[8] M. K. Brown, "The extraction of curved surface features with generic range sensors," Int. J. Robot. Res., vol. 5, pp. 3-18, Spring 1986.

[9] M. L. Hong and L. Kleeman, "Ultrasonic classification and location of 3-D room features using maximum likelihood estimation, I and II," Robotica, vol. 15, pp. 483-491 and pt. 6, pp. 645-652, 1997.

[10] L. Kleeman and H. Akbarally, "A sonar sensor for accurate 3-D target localization and classification," in Proc. IEEE Int. Conf. Robotics Automation, Nagoya, Japan, May 21-27, 1995, pp. 3003-3008.

[11] R. Kuc, "Three-dimensional tracking using qualitative bionic sonar," Robot. Auton. Syst., vol. 11, no. 2, pp. 213-219, 1993.

[12] R. Kuc and M. W. Siegel, "Physically-based simulation model for acoustic sensor robot navigation," IEEE Trans. Pattern Anal. Machine Intell., vol. PAMI-9, pp. 766-778, Nov. 1987.

[13] R. Kuc, "Biologically motivated adaptive sonar system," J. Acoust. Soc. Amer., vol. 100, pp. 1849-1854, Sept. 1996.

[14] _ "Biomimetic sonar recognizes objects using binaural information," J. Acoust. Soc. Amer., vol. 102, pp. 689-696, Aug. 1997.

[15] J. J. Leonard and H. F. Durrant-Whyte, "Mobile robot localization by tracking geometric beacons," IEEE Trans. Robot. Automat., vol. 7, pp. 376-382, June 1991.

[16] P. M. Morse and K. U. Ingard, Theoretical Acoustics. Princeton, NJ: Princeton Univ. Press, 1968.
[17] H. Peremans, K. Audenaert, and J. M. Van Campenhout, "A highresolution sensor based on tri-aural perception," IEEE Trans. Robot. Automat., vol. 9, pp. 36-48, Feb. 1993.

[18] Ultrasonic Components Group, Polaroid Corp., Cambridge, MA, 1990.

[19] A. M. Sabatini, "Statistical estimation algorithms for ultrasonic detection of surface features," in Proc. IEEE/RSJ Int. Conf. Intelligent Robots Systems, Munich, Germany, Sept. 12-16, 1994, pp. 1845-1852.

[20] M. W. Siegel, Personal communication, June 1999.

[21] S. Watanabe and M. Yoneyama, "An ultrasonic visual sensor for threedimensional object recognition using neural networks," IEEE Trans. Robot. Automat., vol. 8, no. 2, pp. 240-249, 1992.

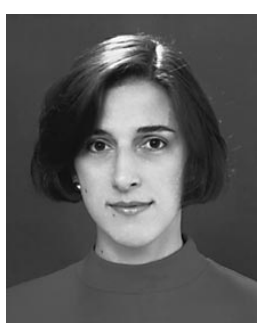

Billur Barshan received B.S. degrees in both electrical engineering and in physics from Boğaziçi University, Istanbul, Turkey, and the M.S. and Ph.D degrees in electrical engineering from Yale University, New Haven, CT, in 1986, 1988, and 1991, respectively.

She was a Research Assistant at Yale University from 1987 to 1991 . From 1991 to 1993 , she was a Postdoctoral Researcher with the Robotics Research Group, University of Oxford, Oxford, U.K. She joined Bilkent University, Ankara, Turkey, in 1993 where she is currently Associate Professor in the Department of Electrical Engineering. She has established the Robotics and Sensing Laboratory in the same department. Her current research interests include intelligent sensing, sensor-based robotics, sonar and inertial navigation systems, and multisensor data fusion.

Dr. Barshan was the recipient of the 1994 Nakamura Prize awarded to the most outstanding paper in 1993 IEEE/RSJ Intelligent Robots and Systems International Conference. She is also the recipient of the 1998 TÜBITTAK Young Investigator Award. 